\title{
The Eclampsia Trial, a model of international collaborative study with worldwide benefits
}

* MD, PhD, MSc, Associate professor of Internal Medicine of Escola Paulista de Medicina, President of the Latinclen Latin American Clinical Epidemiology Network.

$\mathrm{M}$ aternal mortality in Brazil is a major problem. It is about 10 times that of Canada or England. What do these three countries have in common regarding maternal mortality? Hypertension in pregnancy, a major cause of maternal death in developed and developing countries. Among the hypertensive complications of pregnancy, pre-eclampsia and eclampsia are of utmost importance when considering any strategy of maternal death prevention.

A very important issue is the treatment of convulsions in eclampsia. Since the first report of the use of magnesium sulfate in 1906, many other new drugs have been developed and there have been different opinions regarding which the best treatment would be. After 60 years of 'experience' and opinions, preference has been distributed among different types of drugs.

In the US, the large majority of doctors believed the best treatment of fits was magnesium sulfate. About $98 \%$ of the British doctors and many of their colleagues of the Commonwealth preferred diazepam or phenitoin.

Brazilian doctors, being mostly influenced by American medicine, preferred magnesium sulfate; however, diazepam and phenitoin were still considerably used because of the simultaneous influence of British medicine.
The treatment of any severe disease as eclampsia requires a high degree of certainty, whenever possible. A systematic review of literature up to one year ago would result in complete uncertainty as to the best treatment.

The "Collaborative Eclampsia Trial Group Report" of June this year (1) brought the necessary light to this important subject.

Designed as a multicenter collaborative control trial by Dr. Lilia Duley from the University of Oxford, the trial received strong support from South American, African, and Indian researchers. 1,687 patients were randomized and treated in two trial branches: magnesium sulfate versus diazepam (S. America) and magnesium sulfate versus phenitoin (Africa and India). The results showed that the recurrence of fits in the magnesium sulfate groups was $52 \%$ less than in the diazepam group and $67 \%$ less than in the phenitoin-treated groups respectively.

Besides, the study of secondary outcomes suggest important reductions of maternal/fetal morbidity in the patients treated with magnesium sulfate. The authors concluded, based on strong evidence, that magnesium sulfate is the drug of choice for the treatment of convulsions in the patients with eclampsia. These important results led to the establishment, by WHO's Maternal Mortality Committee experts, of the Eclampsia Day, to be observed every June 12 . 
The "Collaborative Eclampsia Trial," which can be considered the most important clinical trial in Obstetrics of this century, was conducted successfully thanks to an association between researchers in a developed country (Oxford University, in the UK) and well-trained researchers in developing countries, where the frequency of maternal complications and deaths is high. The conclusions of this study are useful for all mankind.

There are many other health problems in other areas suitable for applying the "Eclampsia Trial Collaborative Group" study as a model, where the combination of goodwill, expertise, and frequency of outcomes will be integrated to bring light where there is only the heat of medical controversy.

\section{REFERENCES}

1. The Eclampsia Trial Collaborative Group. Which anticonvulsant for women with eclampsia? Evidence from the Collaborative Eclampsia Trial. The Lancet 345:14551463, 1995, 